\title{
A UTILIZAÇÃO DE SOFTWARES DE ANÁLISE DE DADOS QUALITATIVOS SOB O OLHAR DE UMA PESQUISADORA INICIANTE
}

\author{
Dilmeire Sant'Anna Ramos Vosgerau ${ }^{1}$, Ana Paula de Andrade Janz Elias ${ }^{2}$, Thamyres Kinaski \\ Gonçalves $^{3}$ e Beatriz Maria Zoppo ${ }^{4}$ \\ ${ }^{1}$ Universidade Católica do Paraná, Brasil. dilmeire.vosgerau@pucpr.br \\ 2,3 e 4 Pontifícia Universidade Católica do Paraná, Brasil.²anapjanz777@gmail.com \\ 3thamyres.kinaski@live.com, ${ }^{4}$ beazoppo@hotmail.com
}

\begin{abstract}
Resumo. Atualmente, mesmo o pesquisador iniciante em investigações qualitativas pode utilizarse de softwares de análise de dados como auxílio durante todo o processo de investigação. $O$ objetivo deste artigo é apresentar as possibilidades de aprendizados que CAQDAS como o ATLAS.ti, MAXQDA, NVivo e IRAMUTEQ fornecem a pesquisadores iniciantes. Para tanto, foi analisado o relatório final de iniciação científica de uma estudante do Curso de Graduação em Licenciatura em Química da Pontifícia Universidade Católica do Paraná, no qual a estudante tinha por finalidade registrar seu processo de aprendizagem na utilização dos quatro softwares. Foi possível perceber que os QACDAS permitiram à estudante-pesquisadora-iniciante em pesquisa qualitativa debruçar-se na análise dos dados a partir de opções visuais que eles apresentavam. Contudo, ela precisou dedicar um tempo significativo na aprendizagem dos recursos de cada software. Em suas conclusões, é possível perceber que os softwares de análise de dados auxiliam o pesquisador iniciante a comprender o processo de análise qualitativa, contudo, é importante aprender a utilizar essas ferramentas de forma efetiva. Portanto, é de fundamental importância que os pesquisadores, iniciantes no uso de uma ferramenta, prevejam em seu cronograma tempo necessário para esta aprendizagem.
\end{abstract}

Palavras-chave: Sofwares de Análise de Dados; Pesquisa Qualitativa; Pesquisadores Iniciantes.

\section{THE USE OF QUALITATIVE DATA ANALYSIS SOFTWARE UNDER THE EYES OF A BEGINNING RESEARCHER}

\begin{abstract}
Nowadays even the researcher beginning in qualitative investigations can use data analysis software as an aid throughout the investigation process. The purpose of this article is to identify the learning possibilities that the software ATLAS.ti, MAXQDA, NVivo and IRAMUTEQ allow beginning researchers to develop researches that use the approach here mentioned. For this purpose, the Final PIBIC Report of a student of the Undergraduate Degree in Chemistry at the Pontifical Catholic University of Paraná was analyzed and this analysis was based on the analysis processes of a logbook, since the report contained the impressions of the student. It was possible to notice that the QACDAS allowed the student, a researcher beginning in qualitative research, to focus on the analysis of the data from the visual options they presented. In its conclusions, it is possible to notice that the data analysis software helps the beginning researcher to understand the qualitative analysis process, however, it is important to learn how to use these tools effectively. Therefore, it is of fundamental importance that researchers, who are new to the use of a tool, foresee in their schedule the time needed for this learning.
\end{abstract}

Keywords: Data Analysis Software; Qualitative Research; Beginning Researchers.

\section{INTRODUÇÃO}

O pesquisador é considerado como produtor de conhecimentos, sendo assim é responsável por investigar e divulgar dados, bem como por produzir novos aprendizados (O'Leary, 2019); contudo, essa produção de conhecimentos requer rigor no processo de investigação. Desta 
forma, em seu processo de formação, o pesquisador necessita compreender os requisitos necessários para cada etapa da pesquisa a fim de que seja evidenciada a acuidade com que o processo de investigação foi conduzido.

A compreensão de um relato de pesquisa qualitativa se inicia a partir da história de vida dos pesquisadores, suas visões pessoais e em relação a outros, assim como questões éticas e políticas, o que permite ao leitor compreender sob qual perspectiva os dados foram analisados. Em um segundo momento deste relato, "[...] o pesquisador traz para a investigação determinadas teorias, paradigmas e perspectivas teóricas [...]" as quais também estão alinhadas com a visão de mundo do pesquisador (Creswell, 2014, p. 30); em seguida, ele escolhe a abordagem, ou abordagens metodológicas, que irá utilizar na coleta e na análise dos dados, ações que correspondem ao quarto e quinto momentos, respectivamente, desse tipo de investigação (Creswell, 2014).

Para auxiliar os pesquisadores qualitativos na sistematização de suas pesquisas, ou seja, na manipulação e no gerenciamento dos dados coletados, valer-se de alguns recursos como os diferentes tipos de softwares de análise de dados disponíveis hoje é uma possibilidade (Flick, 2009). O uso desses softwares possibilita aos pesquisadores qualitativos dedicar um tempo maior àquilo que é efetivamente essencial ao processo de pesquisa, como a reflexão e a análise dos dados (Creswell, 2016; Corbin \& Strauss, 2016).

Alguns dos CAQDAS (Computer - Assisted Qualitative Data Analysis Software) disponíveis para uso nos dias atuais são: ATLAS.ti'; MAXQDA2; NVivo3; e IRAMUTEQ4. Esses quatro foram investigados por Gonçalves \& Vosgerau (2019), no Programa Institucional de Bolsas de Iniciação Científica (PIBIC) da Pontifícia Universidade Católica do Paraná (PUCPR).

O intuito da pesquisa aqui analisada foi verificar como um pesquisador iniciante em pesquisa qualitativa compreendia as possibilidades de uso dos CAQDAS diante de dados qualitativos em pesquisas educacionais. Nesse viés, este artigo busca aprofundar e ampliar os dados coletados e apresentados por (Gonçalves \& Vosgerau, 2019) no Relatório Final do PIBIC e, para tanto, a seguinte questão foi levantada: quais são as possibilidades de aprendizado que CAQDAS como o ATLAS.ti, MAXQDA, NVivo e IRAMUTEQ fornecem a

\footnotetext{
1 Disponível em https://atlasti.com/. Acesso em 07 de mar. 2020.

2 Disponível em https://www.maxqda.com/. Acesso em 07 de mar. 2020.

3 Disponível em https://www.qsinternational.com/nvivo/nvivo-products. Acesso em 07 de mar. 2020.

4 Disponível em http://www.iramuteq.org/. Acesso em 07 de mar. 2020.
} 
pesquisadores iniciantes em pesquisas qualitativas para a análise e sistematização dos dados coletados durante o desenvolvimento de seus trabalhos?

\section{A PESQUISA QUALITATIVA E OS CAQDAS}

A realidade apresenta grande complexidade e para compreendê-la a pesquisa qualitativa torna-se necessária. Em uma perspectiva de comparação, para entender o que é a pesquisa qualitativa é preciso observar as diferenças entre as pesquisas qualitativa e quantitativa (Cardano, 2017), conforme ilustrado na Tabela 1. Observa-se que o uso de números nos permitem mapear diferentes realidades ou situações, já a informação qualitativa permite interpretar as realidades sociais (Bauer, Gaskell, \& Allum, 2015).

Tabela 1. Principais diferenças entre pesquisa qualitativa e quantitativa (Bauer, Gaskell e Allum, 2015)

\begin{tabular}{lll}
\hline & \multicolumn{2}{c}{ ESTRATÉGIAS } \\
\hline & QUANTITATIVAS & QUALITATIVAS \\
\hline Dados & Números & Textos \\
Análise & Estatística & Interpretação \\
Protótipo & Pesquisa de opinião & Entrevista em profundidade \\
Qualidade & Hard & Soft \\
\hline
\end{tabular}

Independente da abordagem escolhida pelo pesquisador, é interessante ressaltar que as investigações qualitativas tem por base processos de análise e de reflexão da realidade que visam compreender o objeto de estudo (Oliveira, 2013). Na busca por essa compreensão, é possível identificar algumas semelhanças em trabalhos realizados por pesquisadores qualitativos: (1) escolha de uma questão para o desenvolvimento de uma pesquisa; (2) coleta de dados não estruturados para responder à questão escolhida; (3) tratamento dos dados coletados; (4) e análise dos dados para identificar a resposta à questão levantada (Deslauriers; Kérisit, 2012).

A análise dos dados pode ser aprofundada a partir do conhecimento de diferentes teorias e metodologias específicas para essa etapa (Freitas, 2004). A análise é, em diferentes situações, considerada o núcleo central da pesquisa qualitativa (Gibbs, 2009), a qual é apresentada de maneira formal e sistemática ao final da coleta de dados, contudo, é possível afirmar que ela também está presente nos demais estágios da pesquisa.

Por ser um processo sistemático, é possível afirmar que requer organização e sistematização das informações. Existem diferentes maneiras de realizar a organização e sistematização necessárias para o desenvolvimento de uma investigação, entre elas a utilização dos CAQDAS. A utilização de um software na análise de dados possibilita: (1) 
mais velocidade para trabalhar com os dados; (2) aumento da qualidade na pesquisa; (3) ampliação da transparência do processo; (4) a comunicação entre a equipe de pesquisa; (5) além de ter os documentos da pesquisa organizados em um único lugar (Flick, 2009).

Contudo, existem diferentes softwares disponíveis hoje e o pesquisador pode se questionar: qual deles utilizar? A escolha do software deve ser do próprio pesquisadoral irá trabalhar e essa escolha dependerá tanto das necessidades de sua pesquisa quanto de suas preferências pessoais, pois caso o pesquisador não tenha domínio do software, é necessário que seja previsto um período para aprendizagem (Saldaña, 2016).

É necessário ter consciência que o software não realizará nenhum trabalho sozinho, pois apesar de possibilitar: (1) o armazenamento dos dados coletados; (2) a diagramação por meio de mapas conceituais, vinculando códigos e temas; (3) o relacionamento entre temas com localizações geográficas; (4) o trabalho em colaboração com uma equipe de pesquisadores; (5) a compilação das análises realizadas; (6) a geração de relatórios; entre outras funções (Creswell, 2016), ele não substitui as ações do investigador, especialmente as inferências e as reflexões realizadas a partir dos dados coletados em uma pesquisa.

O pesquisador qualitativo que optar por utilizar um CAQDAS deve buscar conhecer as possibilidades dele para auxiliar no processo de gerenciamento dos dados de uma investigação. Ele também precisa conhecer os principais recursos do software para poder utilizar-se de todo o potencial do CAQDAS escolhido, de maneira a agregar valor em sua pesquisa, pois o processo de investigação pode ser melhorado a partir do uso de softwares de análise de dados qualitativos (Creswell, 2010), porém esse aprimoramento depende da postura e do domínio do pesquisador em relação à usabilidade desses recursos.

\section{METODOLOGIA DA PESQUISA}

O relatório do PIBIC, elaborado por (Gonçalves \& Vosgerau, 2019), intitulado "Avaliação da usabilidade de software para a análise de dados qualitativos em pesquisas educacionais", foi analisado como um diário de bordo, no início do ano de 2020 para a execução desse artigo. Esse relatório foi escrito por uma estudante de graduação em Licenciatura em Química da PUCPR, iniciante em processos de investigação qualitativa, com conhecimento básico do uso do computador para fins acadêmico, portanto, uso: (1) do Google para pesquisa; (2) do Word para edição de texto e (3) do Excel para análise e sistematização dos resultados, desconhecendo qualquer software de análise qualitativa de dados. 
Para essa experiência de uso de softwares de análise de dados qualitativos, a orientadora da pesquisa propôs à estudante, denominada nesse estudo como estudante-pesquisadorainiciante, que buscasse uma autoaprendizagem dos softwares ATLAS.ti, MAXQDA, NVivo e IRAMUTEQ a fim de analisar um pacote de dados comum contendo um texto editável (.doc), um texto não editável (.pdf), uma imagem e um vídeo. A Estudante-pesquisadora-iniciante poderia recorrer a qualquer fonte de consulta, exceto curso presencial. A consulta à orientadora deveria ocorrer apenas quando houvesse algum impasse, o qual não permitisse à estudante continuar o processo de autoaprendizagem. Caso a orientadora julgasse que as informações poderiam ser obtidas em manuais ou vídeos disponíveis na Web, a orientação seria apenas de busca. Todo o processo de aprendizagem deveria ser registrado em um diário de bordo.

O diário de bordo é um instrumento no qual o sujeito contempla, por meio de uma narrativa, todas as suas ações e experiências durante um processo de pesquisa (Boszko \& Da Costa Güllich, 2017). A escolha de análise do relatório se deu: por se tratar das primeiras impressões, já sistematizadas, de uma pesquisadora iniciante em pesquisa qualitativa, sobre a utilização de diferentes CAQDAS como ferramenta de auxílio nos processos de gerenciamento e análise dos dados.

Para a análise do relatório da estudante-pesquisadora-iniciante foram definidas quatro categorias, apresentadas no formato de perguntas, para nortear essa investigação, são elas: (1) o que motivou a escolha pelos quatro softwares citados anteriormente?; (2) quais os materiais utilizados pela estudante?; (3) como foi realizada a análise de cada software?; (4) quais foram as conclusões apontadas no relatório?

Após a definição de categorias para análise do relatório, foi realizada leitura e reflexão sobre o texto apresentado, com o intuito de responder a questão norteadora desta pesquisa: quais são as possibilidades de aprendizados que CAQDAS como o ATLAS.ti, MAXQDA, NVivo e IRAMUTEQ fornecem a pesquisadores iniciantes em pesquisas qualitativas para a sistematização dos dados coletatos durante o desenvolvimento de seus trabalhos?

\subsection{Análise dos softwares desenvolvida pela pesquisadora iniciante}

A escolha dos quatro softwares se deu por dois motivos: (1) a professora, orientadora da estudante-pesquisadora-iniciante, já desenvolveu investigações que tiveram auxílio dos softwares ATLAS.ti (Vosgerau, Pocrifka, \& Simonian, 2016) e MAXQDA (Klein \& Vosgerau, 
2018); (2) cursos sobre a utilização dos quatro softwares tem sido oferecido regularmente pela Fundação Carlos Chagas (https://www.fcc.org.br/fcc/cursos-e-seminarios), o que pode ampliar a orientação de uso em pesquisas educacionais. Essa informação, retirada do relatório/diário-de-bordo aqui analisado, responde a primeira categoria levantada nessa pesquisa para análise.

Após a escolha pelos quatro CAQDAS citados anteriormente, a estudante-pesquisadorainiciante realizou um reconhecimento dos softwares escolhidos. Os materiais e o processo de análise utilizados, conforme segunda e terceira categorias levantadas para essa pesquisa, foram descritos no relatório/diário-de-bordo, bem como os caminhos percorridos para acessar os CAQDAS. A estudante-pesquisadora-iniciante comentou sobre as facilidades e dificuldades que teve nos diferentes momentos da pesquisa: no relatório ela aponta uma certa facilidade em baixar os softwares MAXQDA e NVivo, por identificar que, no momento de sua pesquisa, o processo se apresentava de maneira intuitiva, o que já não ocorreu com o ATLAS.ti e o IRAMUTEQ. Durante as instalações dos quatro softwares foram perceptíveis algumas semelhanças, como o idioma em inglês estar presente na maior parte do processo. No fornecimento de dados para fazer a instalação e após a instalação todos já estavam configurados em português. Foi observado que o download do NVivo para um teste foi mais fácil em vista da localização dessa informação no site. Outra facilidade encontrada refere-se à instalação do MAXQDA, o site do software fornece vídeos explicativos no momento em que é selecionada a opção Download da versão teste. Entretanto, para instalar - IRAMUTEQ é necessário assistir a alguns vídeos no YouTube, pois existem alguns detalhes que precisam ser considerados para que ele funcione corretamente. Já o ATLAS.ti necessita de um cadastro prévio e o recebimento de um e-mail com o link de instalação.

Para iniciar o processo de codificação do material selecionado, foi necessário que a estudante-pesquisadora-iniciante assistisse a vídeos tutoriais na internet e que fizesse a leitura de manuais de instrução para compreender os processos que deveriam ser realizados a partir de cada um dos CAQDAS.

Em relação à disponibilidade de material para autoaprendizagem disponível no YouTube, é descrito no relatório que no NVivo, após o processo de instalação, inicia automaticamente um tutorial demonstrando como realizar as codificações. Além disso, existem vídeos em português feitos pelo próprio desenvolvedor sobre a codificação. Os vídeos disponíveis sobre o ATLAS.ti em português, no período de análise (Julho/2018), não contemplavam a 
versão traduzida do software, contudo havia material em inglês e espanhol. Além disso, existem poucos vídeos que expliquem além da codificação do material, ou seja, poucos vídeos sobre como realizar a construção de uma rede semântica e as ferramentas de análise, por exemplo.

No relatório a estudante-pesquisadora-iniciante destaca o aspecto intuitivo dos softwares MAXQDA, NVivo e ATLAS.ti para diversas funções ao contrário do IRAMUTEQ. Durante a análise inicial dos CAQDAS, foram identificados: (1) idiomas suportados nos sites dos fabricantes dos softwares; (2) sistemas operacionais; (3) suporte ao cliente; (4) licenças disponíveis; (5) valor financeiro; (6) características da versão para teste, essas informações ainda contemplam a categoria 3, citada anteriormente.

Essa análise foi detalhada na Tabela 2:

Tabela 2. Análise dos softwares (Gonçalves \& Vosgerau, 2019)

\begin{tabular}{|c|c|c|c|c|c|c|c|c|}
\hline Nome do Softwar - & Site do fabricante & $\begin{array}{l}\text { Idiomas suportados no } \\
\text { site do fabricante }\end{array}$ & Sistema Operacion * & $\begin{array}{l}\text { Ultima } \\
\text { versäo }\end{array}$ & Suporte ao clie ${ }^{*}$ & Licenças disponiveis & Precos $\mathrm{V}$ & Versão para te ${ }^{\text {. }}$ \\
\hline ATLAS.ti & https://atlasticoml & $\begin{array}{l}\text { Inglês, espanhol, japonês, } \\
\text { chinês, francês e alemão. } \\
\text { Apenas em "encomenda e } \\
\text { preço" aparece mais opções } \\
\text { de idioma inclusive } \\
\text { português }\end{array}$ & $\begin{array}{l}\text { Mac, Windows, Andoid } \\
\text { S App, Ipad App }\end{array}$ & 8.3 & $\begin{array}{c}\text { Suporte center, } \\
\text { suporte telefonico } \\
\text { (free), FAQ, } \\
\text { trainers e } \\
\text { consultoria, } \\
\text { newsroom. }\end{array}$ & $\begin{array}{l}\text { Licenças educacionais, } \\
\text { licença para } \\
\text { estudante, licença não } \\
\text { comercial e governamental, } \\
\text { licenças comerciais, licença } \\
\text { de campus }\end{array}$ & $\begin{array}{l}\text { Licenças educacionais: } \\
2.350,81 \text { ( } 1 \text { usuário) } \\
\text { Licença para } \\
\text { estudante } 366,76 \text { (dois } \\
\text { anos); } 188,93 \text { (seis } \\
\text { meses) Licença não } \\
\text { comercial e } \\
\text { governamental: } \\
5.216,08 \text { Licenças } \\
\text { comerciais: } 5.586,10 \text { (1 } \\
\text { usuário) Licença de } \\
\text { campus: orçamento }\end{array}$ & $\begin{array}{l}\text { "Sem limite de } \\
\text { tempo" }\end{array}$ \\
\hline Nvivo & tths://www.qusrinternational.com/nvivo/hom & $\begin{array}{l}\text { Portugês, inglês, espanhol, } \\
\text { francês, alemão, chinês, } \\
\text { japonês e coreano. }\end{array}$ & $\begin{array}{l}\text { Mac, Windows, Andoid } \\
\text { App, Ipad App }\end{array}$ & 12 & $\begin{array}{l}\text { Visão geral, Faqs, } \\
\text { para TI } \\
\text { administradores e } \\
\text { download }\end{array}$ & $\begin{array}{l}\text { Padrão, governamental, sem } \\
\text { fins lucrativos, acadêmica, } \\
\text { estudante. }\end{array}$ & $\begin{array}{l}\text { Padräo: } 5923,66 \text { (Plus); } \\
5182,74 \text { (Pro); } 4441,82 \\
\text { (Mac). Governamental: } \\
5923,66 \text { (Plus); 3626,80 } \\
\text { (Pro); } 4441,82 \text { (Mac). } \\
\text { Sem fins lucrativos: } \\
4145,45 \text { (Pro); } 3108,16 \\
\text { (Mac). Acadêmica: } \\
\text { 2963,68 (Plus); } 2593,22 \\
\text { (Pro); 2222,76 (Mac). } \\
\text { Estudante: } 422,32 \text { (Plus); } \\
\text { 366,76 (Pro); 314,89 } \\
\text { (Mac). }\end{array}$ & $\begin{array}{c}14 \text { dias } \\
\text { gratuitamente }\end{array}$ \\
\hline MaxQda & https://www.maxada.com/ & $\begin{array}{l}\text { Portugês, inglês, espanhol, } \\
\text { francês, alemão, chinês, } \\
\text { japonês, italiano, polonês, } \\
\text { etc. }\end{array}$ & Mac, Windows & 12 & $\begin{array}{l}\text { Tutorial em forma } \\
\text { de video, manuais } \\
\text { e guias em forma } \\
\text { de texto, cursos } \\
\text { online e presenciais } \\
\text { de MAXQDA, } \\
\text { instrutores } \\
\text { MAXQDA no Brasil }\end{array}$ & $\begin{array}{l}\text { Estudantes, pesquisadores, } \\
\text { professores ou instituições. }\end{array}$ & $\begin{array}{l}\text { Estudantes: } 159,30 \text { (6 } \\
\text { meses); } 307,48 \text { ( } 24 \\
\text { meses); } 340,82 \text { (Pro, } 12 \\
\text { meses); } 426,03 \text { (Pro, } 24 \\
\text { meses). Pesquisadores, } \\
\text { professores ou } \\
\text { instituiçöes: } \\
\text { "orcamento". }\end{array}$ & $\begin{array}{l}14 \text { dias } \\
\text { gratuitamente }\end{array}$ \\
\hline IRAMUTEq & hittp:/liramuteq orga/ & Francês & Linux, Windows, Macos & 0.7 alpha 2 & & & & \\
\hline
\end{tabular}

Em resposta a quarta categoria aqui levantada, foi possível identificar no relatório/diário-debordo que os quatro softwares analisados apresentam diferentes recursos, para que o pesquisador possa ter uma visão geral da codificação realizada e conseguir por meio deles realizar inferências, ou seja, um segundo ciclo de codificação, conforme denomina Saldaña (2016). Como exemplo desses recursos tem-se: (1) o recurso de nuvem de palavras 
(Gonçalves \& Vosgerau, 2019); (2) a rede no ATLAS.ti; (3) o mapa de projeto no NVivo; (4) a similitude no IRAMUTEQ; e (5) o maxmapa no MAXQDA.

Após a instalação dos CAQDAS e da análise dos materiais indicados sobre pesquisa qualitativa e sobre os próprios CADQDAS estudados, a estudante-pesquisadora-iniciante optou por apresentar os resultados das análises realizadas de forma visual.

Para a aprendizagem do software NVivo, a estudante-pesquisadora-iniciante optou por organizar os dados coletados em sua pesquisa, conforme é possível observar na Figura 1.

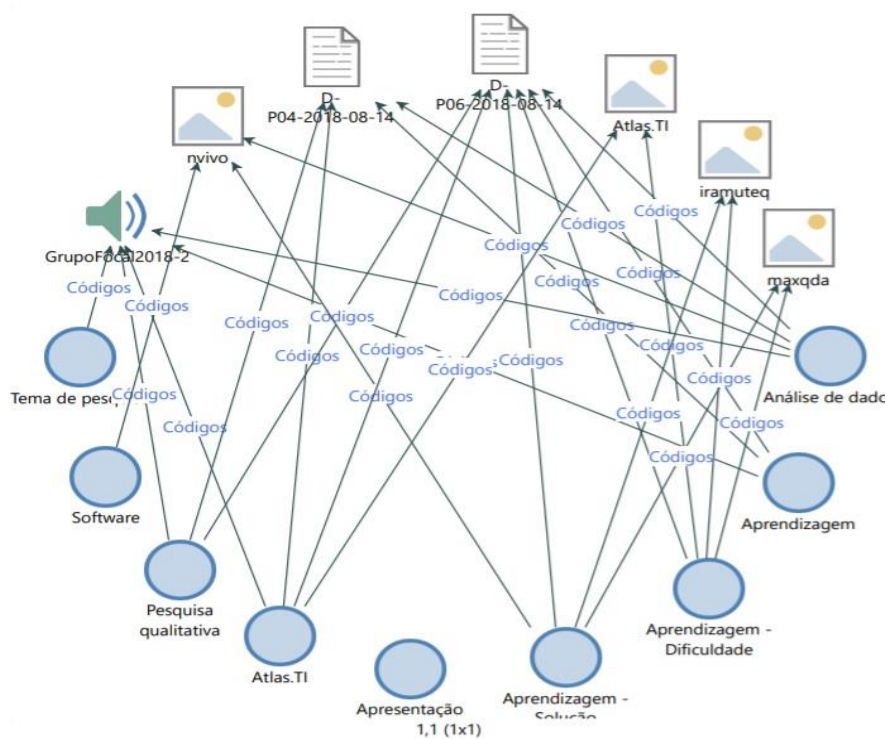

Figura 1. Mapa do projeto PIBIC no software NVivo (Gonçalves \& Vosgerau, 2019, p. 09).

Nessa figura é apontado quais são as possibilidades que a estudante-pesquisadora-iniciante encontrou em cada um dos softwares bem como os processos de aprendizagem pelos quais ela percorreu, é possível identificar isso ao observar que ela nomeou alguns itens do mapa por 'Aprendizagem'; 'Aprendizagem-Solução'; 'Aprendizagem-Dificuldade'. Dessa maneira verifica-se que a opção por essa representação gráfica no software NVivo também trouxe uma possibilidade de a estudante-pesquisadora-iniciante organizar o trabalho que estava desenvolvendo ao longo de seu projeto PIBIC.

Já na rede do software ATLAS.ti (Figura 2) a opção de representação gráfica foi no intuito de comparar e relacionar as opções de análise dos CAQDAS, bem como no intuito de realizar algumas inferências sobre os processos da pesquisa qualitativa, especialmente o de codificação de dados. 


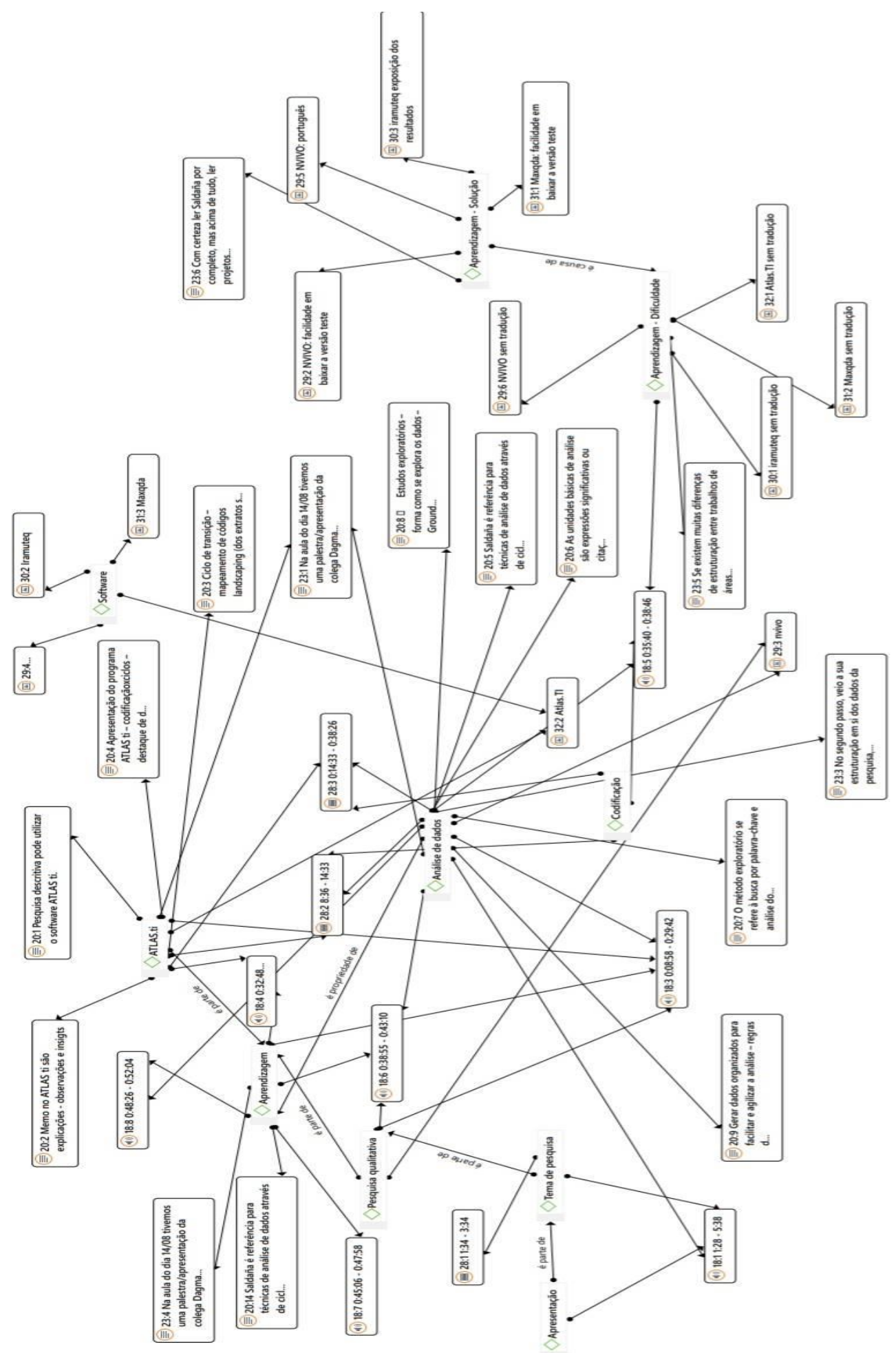

Figura 2. Resultados em forma de rede no ATLAS.ti (Gonçalves \& Vosgerau, 2019, p. 10)

$\mathrm{Na}$ análise de similitude gerada no software IRAMUTEQ (Figura 3), a estudantepesquisadora-iniciante pontuou algumas informações que considerou importante no 
percurso da pesquisa, tais como a coleta dos dados, o processo de análise e o método da pesquisa. Pela imagem é possível verificar que ainda não houve uma sistematização efetiva realizada por essa pesquisadora para o processo de uma pesquisa qualitativa, mas existe uma compreensão inicial, a qual pode ser aprofundada com o passar do tempo dedicado a pesquisa.

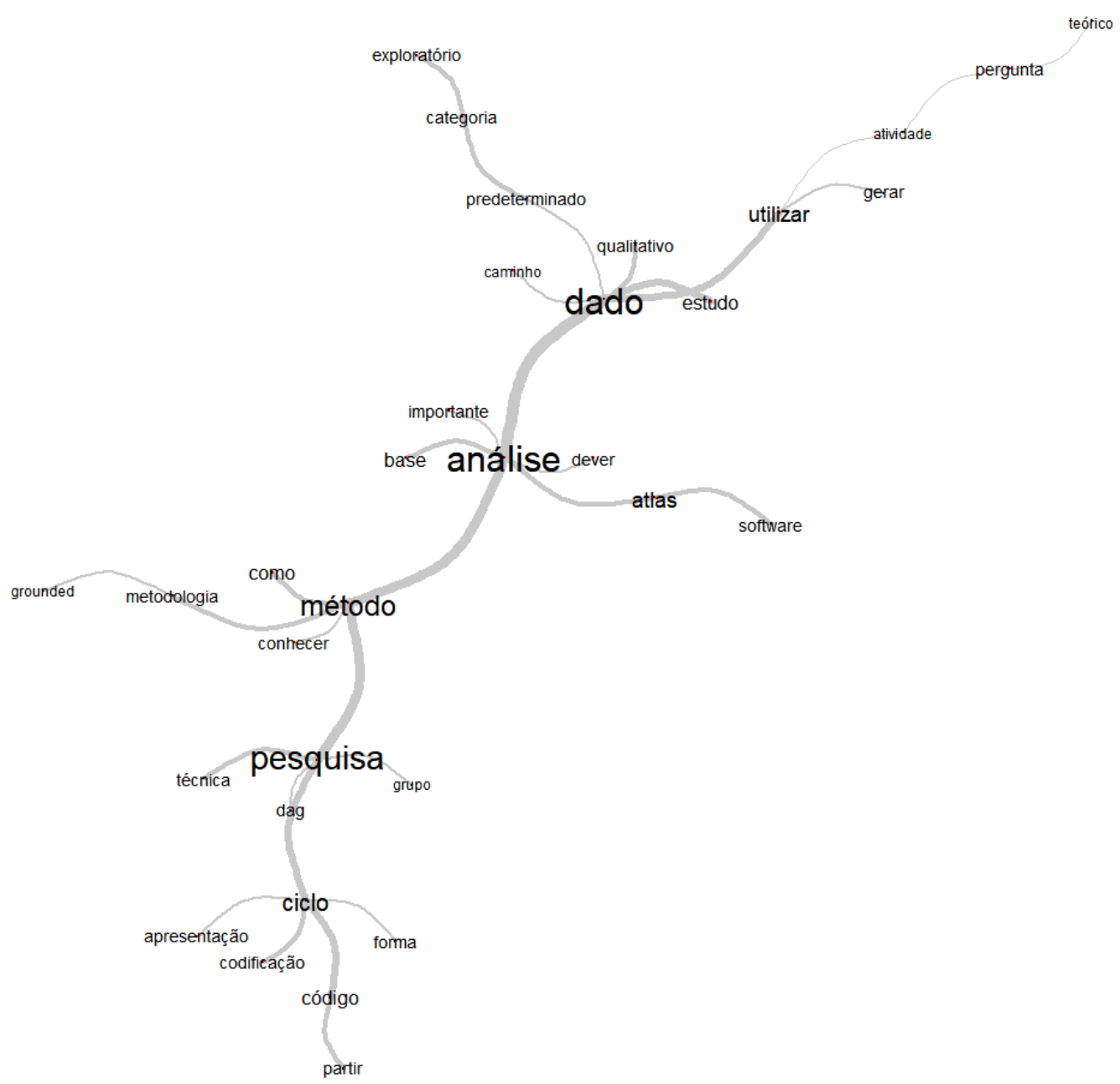

Figura 3. Análise de Similitude gerado pelo IRAMUTEQ (Gonçalves \& Vosgerau, 2019, p. 11).

No Maxmapa desenvolvido e colocado no relatório/diário-de-bordo (Figura 4), é possível verificar que houve a opção por apresentar os processos de instalação dos softwares, as dificuldades e também a solução escolhida pela estudante-pesquisadora-iniciante. 


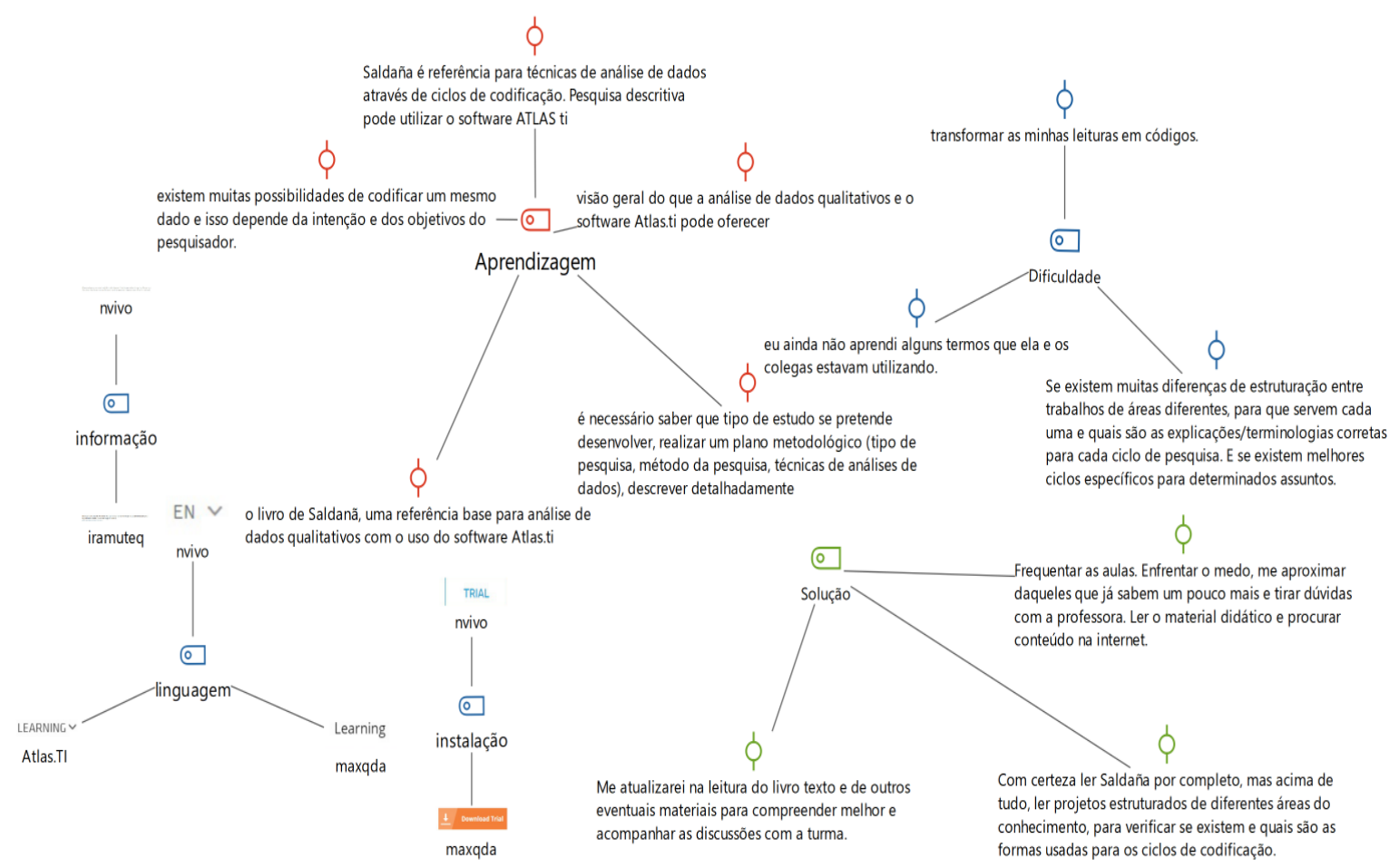

Figura 4. Maxmapa gerado pelo MAXQDA (Gonçalves \& Vosgerau, 2019, p. 12)

Foi importante para a estudante-pesquisadora-iniciante realizar uma pesquisa bibliográfica para compreender o que era efetivamente uma pesquisa qualitativa, pois "como estudante de Licenciatura em Química havia uma carência sobre os conhecimentos de pesquisa qualitativa e análise dos dados" (Gonçalves \& Vosgerau, 2019, p. 13).

Quanto às possibilidades de uso dos CAQDAS, a pesquisadora-iniciante aponta que teve mais facilidade na utilização do software MAXQDA, porque para ela "a interface do software é organizada e intuitiva, o que facilita e diminui o tempo destinado a aprendizagem da utilização do software" (Gonçalves \& Vosgerau, 2019, p. 13).

O fato de os softwares MAXQDA e NVivo disponibilizarem poucas semanas para o uso de suas versões teste dificultou o trabalho integral da pesquisadora, pois, como ela precisou reservar um tempo para aprender a utilizar os recursos, a análise acabou sendo superficial e não foi possível aprofundá-la efetivamente.

Após ter tipo a experiência de trabalhar com os quatro CAQDAS, a estudante-pesquisadorainiciante concluiu que apesar de ter menos vídeos disponíveis em português para 
autoaprendizagem: "para uma análise mais completa e de fácil entendimento o ATLAS.ti é o software ideal [...]" (Gonçalves \& Vosgerau, 2019, p. 14), pois ele apresenta mais recursos e facilita as relações das análises desenvolvidas; e ainda "o uso de softwares é uma maneira de facilitar o tratamento de dados, além de proporcionar uma visão mais objetiva e melhorar a qualidade da pesquisa" (Gonçalves \& Vosgerau, 2019, p. 15).

\section{CONSIDERAÇÕES FINAIS}

O pesquisador iniciante em pesquisa qualitativa necessita, em um primeiro momento, compreender as peculiaridades de uma pesquisa nesse formato. Para que consiga reservar mais tempo para o que efetivamente é importante, como reflexões e inferências a partir do material coletado.

Conforme foi possível identificar pela análise do Relatório Final do PIBIC, escrito pela estudante-pesquisadora-iniciante, faz-se necessário um conhecimento prévio dos recursos de cada software que se quer utilizar no momento da pesquisa. Esse conhecimento prévio possibilitará que, posteriormente, o foco do pesquisador esteja efetivamente nos dados coletados.

Foi possível identificar que o foco das aprendizagens que cada um dos softwares permitiu à estudante-pesquisadora-iniciante estava diretamente relacionado à organização dos dados para a análise e para a apresentação visual desses dados.

Contudo, o fato de os softwares ATLAS.ti, NVivo e MAXQDA possuírem algumas limitações na versão gratuita, não foi possível identificar todo o potencial para o aprendizado de pesquisadores iniciantes que esses recursos possibilitam.

Nesse viés, fazem-se necessárias novas pesquisas quanto à usabilidade $\mathrm{e}$ às potencialidades de cada software em pesquisas qualitativas, de maneira a auxiliar o trabalho do pesquisador.

Essas novas investigações podem ser individuais ou até mesmo comparativas, pois é o pesquisador que escolherá o software que melhor se adeque a suas expectativas e a sua individualidade, bem como às particularidades de cada pesquisa. 


\section{REFERÊNCIAS}

Bauer, M. W., Gaskell, G., \& Allum, N. C. (2015). Qualidade, quantidade e interesses do conhecimento - Evitando confusões. In M. W. Bauer \& G. Gaskell (Eds.), Pesquisa qualitativa com texto, imagem e som: um manual prático (2nd ed., p. 23). Petrópolis: Vozes.

Bogdan, R. C., \& Biklen, S. K. (1994). Investigação qualitativa em educação (2nd ed.). Portugal: Porto editora.

Boszko, C., \& Da Costa Güllich, R. I. (2017). O Diário De Bordo Como Instrumento Formativo No Processo De Formação Inicial De Professores De Ciências E Biologia. Revista Bio-Grafía Escritos Sobre La Biología y Su Enseñanza, 9(17), 55. https://doi.org/10.17227/20271034.vol.9num.17bio-grafia55.62

Cardano, M. (2017). Manual de pesquisa qualitativa: a contribuição da teoria da argumentação. Petrópolis: Vozes.

Corbin, J., \& Strauss, A. (2016). Basics of qualitative research: techniques and procedures for developing grounded theory. Thousand Oaks: Sage.

Creswell, J. W. (2010). Projeto de pesquisa: Métodos Qualitativo, Quantitativo e Misto (3. ed.). Porto Alegre: Artmed.

Creswell, J. W. (2014). Investigação qualitativa e projeto de pesquisa (3rd ed.). Porto Alegre: Penso.

Creswell, J. W. (2016). 30 Essential skills for the qualitative researcher. Thousand Oaks, California: Sage Publications.

Deslauriers, J.-P., \& Kérisit, M. (2012). O delineamento da pesquisa qualitativa. In J. Poupart, J.-P. Deslauriers, L.-H. Groulx, A. Laperriére, Ro. Mayer, \& Á. Pires (Eds.), A pesquisa qualitativa: enfoques epistemológicos e metodológicos (3rd ed., pp. 127-153). Petrópolis: Vozes.

Flick, U. (2009). Introdução à pesquisa qualitativa (3rd ed.). Porto Alegre: Artmed.

Freitas, N. K. (2004). Pesquisa qualitativa e formação do pesquisador: questões teórico-metodológicas. 22, 2732.

Gibbs, G. (2009). Análise de dados qualitativos (1st ed.). Porto Alegre: Artmed.

Gonçalves, T. K., \& Vosgerau, D. S. R. (2019). Avaliação da usabilidade de software para a análise de dados qualitativos em pesquisas educacionais. Pontifícia Universidade Católica do Paraná, Curitiba.

Klein, E. L., \& Vosgerau, D. S. R. (2018). Possibilidades e desafios da prática de aprendizagem colaborativa no ensino superior. Educação (UFSM), 43(41), 667-698. Retrieved from https://periodicos.ufsm.br/reveducacao/article/view/29300

O’Leary, Z. (2019). Como fazer seu projeto de pesquisa: guia prático. Petrópolis: Vozes.

Oliveira, M. M. de. (2013). Como fazer pesquisa qualitativa (5th ed.). Petrópolis: Vozes.

Saldaña, J. (2016). The coding: manual for qualitative researchers (3rd ed.). Thousand Oaks, California: SAGE Publications Inc.

Vosgerau, D. S. R., Pocrifka, D. H., \& Simonian, M. (2016). Etapas da análise de conteúdo complementadas por ciclos de codificação: possibilidades a partir do uso de software de análise qualitativa de dados. Atas CIAIQ2016, 1(0), 789-798. https://doi.org/10.17013/risti.19.93 